\title{
YWHAZ promotes ovarian cancer metastasis by modulating glycolysis
}

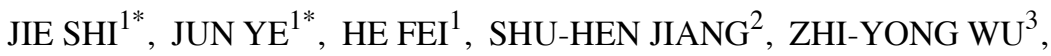 \\ YA-PING CHEN ${ }^{1}$, LI-WEN ZHANG ${ }^{1}$ and XIAO-MEI YANG ${ }^{2}$ \\ ${ }^{1}$ Department of Obstetrics and Gynecology, The Fifth People's Hospital of Shanghai, Fudan University; \\ ${ }^{2}$ State Key Laboratory of Oncogenes and Related Genes, Shanghai Cancer Institute, Renji Hospital, \\ Shanghai Jiao Tong University School of Medicine, Shanghai 200240; ${ }^{3}$ Department of Gynecology, \\ Obstetrics and Gynecology Hospital, Fudan University, Shanghai 200011, P.R. China
}

Received July 1, 2018; Accepted December 5, 2018

DOI: 10.3892/or.2018.6920

\begin{abstract}
Ovarian cancer is one of the three most deadly gynecological cancers, with the highest mortality rate. As the main cause of death, metastasis is considered to be a crucial factor that reduces the survival time of ovarian carcinoma patients. YWHAZ (also known as 14-3-3 ) influences diverse vital cellular processes such as metabolism, signal transduction, apoptosis and cell cycle regulation. In the present study, we determined that YWHAZ is upregulated in ovarian cancers in contrast to normal tissues by immunohistochemical staining. High YWHAZ expression was found to be associated with TNM stage and metastasis-free prognosis of ovarian cancer patients. Silencing of YWHAZ inhibited the proliferation and facilitated serum starvation-induced apoptosis of ovarian cancer cells. Cell migration was also suppressed by YWHAZ silencing. Furthermore, using an in vivo metastatic model, we found that YWHAZ silence also inhibited ovarian cancer metastasis in vivo. Notably, glycolysis was clearly inhibited in YWHAZ-silenced ovarian cancer cells as determined by lactate production assay and Seahorse XF analysis. YWHAZ also regulated the $\mathrm{PI} 3 \mathrm{~K} / \mathrm{Akt1} /$ vimentin signaling pathway in ovarian cancer cells as detected by western blot analysis. Taken together, our results demonstrated that YWHAZ plays
\end{abstract}

Correspondence to: Dr Li-Wen Zhang, Department of Obstetrics and Gynecology, The Fifth People's Hospital of Shanghai, Fudan University, 108 Heqing Road, Minhang, Shanghai 200240, P.R. China E-mail: 13321837079@163.com

Dr Xiao-Mei Yang, State Key Laboratory of Oncogenes and Related Genes, Shanghai Cancer Institute, Renji Hospital, Shanghai Jiao Tong University School of Medicine, 800 Dongchuan Road, Shanghai 200240, P.R. China

E-mail: xmyang@shsci.org

*Contributed equally

Key words: epithelial ovarian cancer, YWHAZ, 14-3-3६, glycolysis, PI3K, AKT1 an important role in the progression of ovarian cancer and can be used as a potential target for the diagnosis and treatment of epithelial ovarian cancer.

\section{Introduction}

According to the National Cancer Institute (NCI) report, approximately 22,240 new cases of ovarian cancer will be diagnosed in the US in 2018, and 14,070 patients will die of this disease (1). For stage I, II III and IV epithelial ovarian cancer patients, the 5-year relative survival rates are $89,70,36$ and $17 \%$, respectively, and the 10 -year relative survival rates are $84,59,23$ and $8 \%$, respectively (2).

In addition to conventional cytoreductive surgery and platinum-based chemotherapy, tumor targeted therapy has also been used in patients with advanced ovarian cancer, but many patients still relapse after treatment. The survival rate has still not improved, and the mechanisms of occurrence, development, invasion and metastasis in ovarian cancer patients remain unclear. Thus, a significant unmet medical need exists for the development of efficacious therapies that can improve the survival rate of these patients (3).

Therefore, exploring the biological characteristics of tumor cells that are common but differ from normal cells and seeking a specific intervention for this characteristic is the key to improve the efficacy of tumor therapy.

Metabolic reprogramming is a hallmark of cancer (4). Tumor cells exhibit an altered metabolic phenotype characterized by increased glycolysis and diminished oxidative phosphorylation that is called 'aerobic glycolysis' or the 'Warburg effect' $(5,6)$. Increased glycolysis in cancer cells switches cellular metabolic flux to produce more biological building blocks, thereby sustaining rapid proliferation (7). Intermediates of the tricarboxylic acid cycle and metabolites relating to energy metabolism, amino acids, and gut microbial metabolism are perturbed in breast and ovarian cancer cells compared with normal cells (8). As ovarian cancer progresses, complete oxidation of glucose and fatty acids is significantly reduced and occurs concurrently with increases in lactate excretion and $3 \mathrm{H}$-deoxyglucose uptake by late-stage cancer cells, shifting the cells towards a more glycolytic phenotype; 
this notion is confirmed by metabolic changes during ovarian cancer progression (9). A recent study demonstrated that inhibition of glycolysis and glutaminolysis may be a promising therapeutic strategy for the treatment of ovarian cancer (10).

YWHAZ (also known as 14-3-3zeta, 14-3-3 $\zeta$ ) is a member of the 14-3-3 family proteins influencing diverse vital cellular processes, such as metabolism, signal transduction, apoptosis and cell cycle regulation. Clinical studies confirm that overexpression of YWHAZ is negatively correlated with prognosis in multiple tumors, including adenocarcinoma of the esophago-gastric junction (AEG) (11), breast cancer (12), head and neck (13) and hepatocellular carcinoma (14).

YWHAZ may contribute to the development of early stage cancers and promote the transition to invasive cancers (15). High YWHAZ expression in the primary tumor was found to be significantly associated with earlier time to recurrence and distant metastasis (16). As a phospho-serine/-threonine binding protein, YWHAZ plays important roles in the genesis and development of cancer via binding to different partners, such as MEK/ERK (7), HIF-1 $\alpha$ (13) or TGF- $\beta /$ Smads (15). Thus, inhibiting YWHAZ (14-3-3 $\zeta$ ) functional activities might prove to be of potential therapeutic utility. The development of inhibitors for the 14-3-3 family has been somewhat successful, but achieving selectivity for specific family members has proven to be challenging. Therefore, in 14-3-3\}-overexpressing tumors, intercepting downstream effector targets of 14-3-3 may be an effective therapy. Importantly, studies have demonstrated that 14-3-3 regulates cellular metabolic processes. For example, 14-3-3 binding to the cardiac isoform of 6-phosphofructo-2-kinase/fructose-2,6-bisphosphatase (PFK-2) suggests that 14-3-3 is involved in regulating glycolysis and metabolism(17).Inaddition, 14-3-3 ' prevents BAD-BAXmitochondrial localization and protects $\beta$ cells from multiple stressors (18). Increasing 14-3-3 $\zeta$ expression in human mammary epithelial cells (hMECs) upregulated LDHA expression, elevated glycolytic activity and promoted early transformation. Knockdown of LDHA in 14-3-3\}-overexpressing hMECs significantly reduced glycolytic activity and inhibited transformation (7).

At present, whether YWHAZ regulates epithelial ovarian cancer (EOC) metastasis through glycolysis has not yet been studied. Kim et al evaluated the prognostic value of quantitative metabolic parameters measured on F-18 FDG PET/CT (FDG PET/CT) at the time of the first relapse in patients with relapsed EOC (19) and motivated us to explore whether glycolysis and its downstream effectors are involved in YWHAZ-mediated ovarian cancer cell migration.

In the present study, it was found that YWHAZ is upregulated in ovarian cancer and high expression of YWHAZ predicts poor survival in ovarian cancer patients. Furthermore, we revealed that YWHAZ promoted ovarian cancer metastasis by activating the PI3K/Akt1/vimentin signaling pathway and upregulating glycolysis. These data could help us understand the effect and the underlying mechanism of YWHAZ in ovarian cancer metastasis.

\section{Materials and methods}

Cell culture and reagents. Human ovarian carcinoma cell lines ES-2, SKOV3, 293T, HEY, COV318 and OVCAR3 were provided by the Shanghai Cancer Institute (Shanghai, China).
ES-2 and SKOV3 cells were used in assessing the function of YWHAZ on cellular behaviors and the activation of glycolysis. Other cell lines were only used for the detection of YWHAZ expression levels. All cells were cultured according to the instructions of the American Type Culture Collection (ATCC; Manassas, VA, USA). Antibodies used in this study included YWHAZ (cat. no. ab51129; Abcam, Cambridge, MA, USA), AKT (cat. no. ab18785; Abcam), p-AKT1 (cat. no. ab133458; Abcam), PI3K-p85 $\alpha$ (cat. no. 13666), vimentin (cat. no. 5741), LDHA (cat. no. 2012), PFKP (cat. no. 5412), tubulin (cat. no. 2146; all were from Cell Signaling Technology, Danvers, MA, USA).

RNA isolation and real-time $q P C R$. RNA isolation and real-time qPCR were performed as previously described (6). Total RNA of ES-2 and SKOV3 cells was extracted using TRIzol reagent (Takara Bio Inc., Shiga, Japan). cDNA was synthesized by PrimeScript RT-PCR kit (Takara Bio Inc.) based on the manufacturer's protocols. Quantitative real-time PCR was run with SYBR Premix Ex Taq (Takara Bio Inc.) on the 7500 Real-time PCR system (Applied Biosystems; Thermo Fisher Scientific, Inc., Waltham, MA, USA). The cDNA was diluted at 1:20 before use. Primers used for YWHAZ were: 5'-AGGAGCCCGTAGGTCATCTT-3' (forward) and 5'-TGC TTGTGAAGCATTGGGGA-3' (reverse). Primers used for 18S were: 5'-TGCGAGTACTCAACACCAACA-3' (forward) and 5'-GCATATCTTCGGCCCACA-3' (reverse). The cycling settings were as follows: $95^{\circ} \mathrm{C}$ for $10 \mathrm{~min} ; 95^{\circ} \mathrm{C}$ for $10 \mathrm{sec}$, $60^{\circ} \mathrm{C}$ for $30 \mathrm{sec}$ for 45 cycles; $40^{\circ} \mathrm{C}$ for $30 \mathrm{sec}$. Expression level and fold change were calculated using the $\Delta \Delta \mathrm{Cq}$ method (20).

Plasmid construction and transfection. Two stable plasmids for opposite functions were constructed. For stable YWHAZ interference, short hairpin RNA (shRNA) targeting YWHAZ or control shRNA was constructed into the PLKO.1 vector (Invitrogen; Thermo Fisher Scientific, Inc.). The sequences of the shRNAs were designed based on the sequence of siRNA-1 of YWHAZ (si1). For stable overexpression of YWHAZ, the cDNA encoding YWHAZ was subcloned into the pCDH-CMV-MCS-EF1-Puro (CD510B-1) vector to generate the YWHAZ-overexpressing plasmid. Then C510B-1-YWHAZ-Linker-HA plasmids which had been constructed were packaged as required. Virus packaging was performed in $293 \mathrm{~T}$ cells after cotransfection of constructed plasmids with Lipofectamine 2000 (Invitrogen; Thermo Fisher Scientific, Inc.; cat. no. 11668-019). Viruses were harvested at 48 and $72 \mathrm{~h}$ after transfection, and virus titers were determined. ES-2 and SKOV3 cells, two target cell lines, were infected with $1 \times 10^{8}$ recombinant lentivirus-transducing units plus $8 \mu \mathrm{g} / \mathrm{ml}$ polybrene (Sigma-Aldrich, Shanghai, China). Cells were selected and maintained in the presence of $2 \mu \mathrm{g} / \mathrm{ml}$ puromycin. The transfection efficiency was assessed by RT-PCR and western blotting at both the mRNA and protein levels. Subsequent experiments were performed after 2 weeks of puromycin selection.

siRNA transfection. Lipofectamine RNAiMAX transfection reagent (Invitrogen Thermo Fisher Scientific, Inc.) was used for transfecting the designed small interfering RNAs (siRNAs) into ES-2 and SKOV3 cells. The transfection steps were 
carried out following the manufacturer's instructions. Diluted siRNA was added into diluted Lipofectamine RNAiMAX reagent for a 5-min incubation at room temperature. Then, the siRNA-lipid complex was added to the targeted cells. The final siRNA applied per well was 25 pmol in one 6-well plate. The sequences were: GGAGAUUACUACCGUUACUdTdT (hs-YWHAZ-si-1), GAGCUGAAUUAUCCAAUGAdTdT (hs-YWHAZ-si-2) and CGUCUCAAGUAUUGAACAAdT dT (hs-YWHAZ-si-3). Non-targeting scrambled siRNA was used as a control in the transfection. The silencing effects were verified by qRT-PCR and western blot analysis.

Cell proliferation assay. Cell proliferation assay was performed as previously described (21). ES-2 and SKOV3 cells were seeded in 96-well plates at the concentration of 5,000 cells in $100 \mu \mathrm{l}$ complete medium/well before transfection. After the cells were attached, transient transfection was performed according to the protocols. At 24, 48 and $72 \mathrm{~h}$ after siRNA transfection, $10 \mu \mathrm{l}$ of Cell Counting Kit-8 (CCK-8) reagents (Share-bio, Shanghai, China) was added to each well for $1 \mathrm{~h}$ of incubation. The absorbance was measured at $450 \mathrm{~nm}$ using a microplate reader. All experiments were performed in triplicate.

Cell migration assay. ES-2 and SKOV3 cell migration was determined using $8-\mathrm{mm}$ pore size cell culture inserts within a 24-well plate (Corning Inc., Corning, NY, USA) based on the protocols. Cells $\left(2 \times 10^{4}\right)$ in $100 \mu 1$ serum-free medium were added into the upper chamber with $700 \mu \mathrm{l}$ serum medium in the lower chamber and incubated for $24 \mathrm{~h}$. The migrated cells were immobilized with $4 \%$ paraformaldehyde and stained with $0.1 \%$ crystal violet. The quantity of migrated cells was counted under a light microscope in six random fields. Each experiment was performed in triplicate.

Apoptosis assay. ES-2 and SKOV3 cells were seeded on 6-well plates. Cell death was assessed by double-labelling with Annexin V-FITC and propidium iodide (PI) according to the protocols. After induction of apoptosis by serum-free media, the required cells were harvested and washed in cold phosphate-buffered saline (PBS) followed by resuspending the cells in Annexin-binding buffer. After addition of $5 \mu \mathrm{l}$ of Annexin V to each $100 \mu \mathrm{l}$ of cell suspension, the cells were incubated for $15 \mathrm{~min}$ at room temperature. Then propidium iodide (PI) was added 5 min before detection on a flow cytometer (Becton-Dickinson; BD Biosciences, San Jose, CA, USA).

Western blotting. Total protein was extracted from ES-2 and SKOV3 cells using a total protein extraction buffer (Beyotime Institute of Biotechnology, Haimen, China). BCA Protein Assay kit was used to measure the protein concentration. Total protein $(50 \mu \mathrm{g})$ was added into each sample well. Then the same amounts of proteins were separated by $12 \%$ SDS-PAGE and transferred to $\mathrm{NC}$ membranes. The membranes were blocked with $5 \%$ non-fat dry milk for $1 \mathrm{~h}$ and washed twice with TBS wash buffer. Then the membranes were incubated at $4^{\circ} \mathrm{C}$ overnight with the following primary antibodies: YWHAZ (dilution 1:1,000), p-Akt1 (dilution 1:1,000), tubulin (dilution 1:2,000), PI3K (dilution 1:2,000), vimentin (dilution 1:1,000), LDHA (dilution 1:2,000) and PFKP (dilution 1:1,000). After washing with TBS wash buffer, HRP-conjugated anti-rabbit (cat. no. ab205718; Abcam) and anti-mouse (cat. no. ab205719; Abcam) secondary antibodies were diluted (dilution 1:10,000) and added for incubation for $1 \mathrm{~h}$ at room temperature. The protein bands were detected by an ECL kit (Share-bio).

$H \& E$ staining. For $H \& E$ staining, the tissue samples were stained with hematoxylin and eosin. The staining procedures were performed routinely. Firstly, the sections which were pretreated were rinsed in to distilled water. Then the nuclei were stained with hematoxylin for $10 \mathrm{~min}$. After washing in running tap water for $5 \mathrm{~min}$, the sections were differentiated with $0.3 \%$ acid alcohol for $1 \mathrm{~min}$. For bluing, the sections were inserted in $0.2 \%$ ammonia water later following by a 5 -min wash with water. Then the sections were counterstained in eosin for $1 \mathrm{~min}$. Finally, dehydration, clearing and mounting of the sections were performed.

In vivo metastatic model. Mice were supplied by and cultivated in the SPF Animal Laboratory of East China Normal University according to the protocols approved by the Animal Care Commission and received humane care according to the criteria outlined in the 'Guide for the Care and Use of Laboratory Animals' prepared by the National Academy of Sciences and published by the National Institutes of Health. A total of 20 female BALB/C nude mice (6-weeks old) were divided into four groups for the metastasis experiments. They lived in standard housing conditions with $10 \mathrm{~h}$ of light and $14 \mathrm{~h}$ of dark cycle. The indoor temperature was maintained at $26^{\circ} \mathrm{C}$ and the relative humidity was maintained at $40-60 \%$. The particles in the air did not exceed $>0.3 \mu \mathrm{m}$. The food and water were sterilized by special treatment. We treated ES2 and SKOV3 cell lines with stable transfection in advance. After a 2-week puromycin selection, $2 \times 10^{6}$ SKOV3 or ES2 cells expressing Lenti-shcontrol or Lenti-shYWHAZ were injected into the tail vein of nude mice ( $n=5$ each group). The mice were sacrificed by neck dislocation 4 weeks later according to common protocols of the metastasis assay used in numerous literatures and the number of metastatic nodules in the lung was counted under an inverted microscope (Olympus Corporation, Tokyo, Japan). The harvested lungs were fixed and stained by H\&E for histologic assessment.

Lactate production assay. Lactate production was detected as previously described using the Lactate Assay kit (cat. no. ABIN411683; BioVision Inc., Mountain View, CA, USA) (6). Lactate production was measured at the absorbance $(570 \mathrm{~nm})$ with a microplate reader.

Seahorse XF analysis. The extracellular acidification rate (ECAR) of control or YWHAZ-knockdown ovarian cancer cells was assayed on the Seahorse Extracellular flux analyzer (Seahorse Bioscience, Billerica, MA, USA) to evaluate glycolysis according to the manufacturer's instructions (6). SKOV3 and ES2 cells were seeded on a XF96-well plate at a density of $4 \times 10^{4} /$ well and allowed to attach overnight, followed by transfection with the negative-control siRNA or siRNAs targeting YWHAZ. After $24 \mathrm{~h}$, cells were incubated in a non- $\mathrm{CO}_{2}$ $37^{\circ} \mathrm{C}$ incubator with unbuffered medium for $1 \mathrm{~h}$ followed by a sequential injection of $10 \mathrm{mM}$ glucose, $1 \mu \mathrm{M}$ oligomycin (Sigma-Aldrich; Merck KGaA, Darmstadt, Germany) and 
A

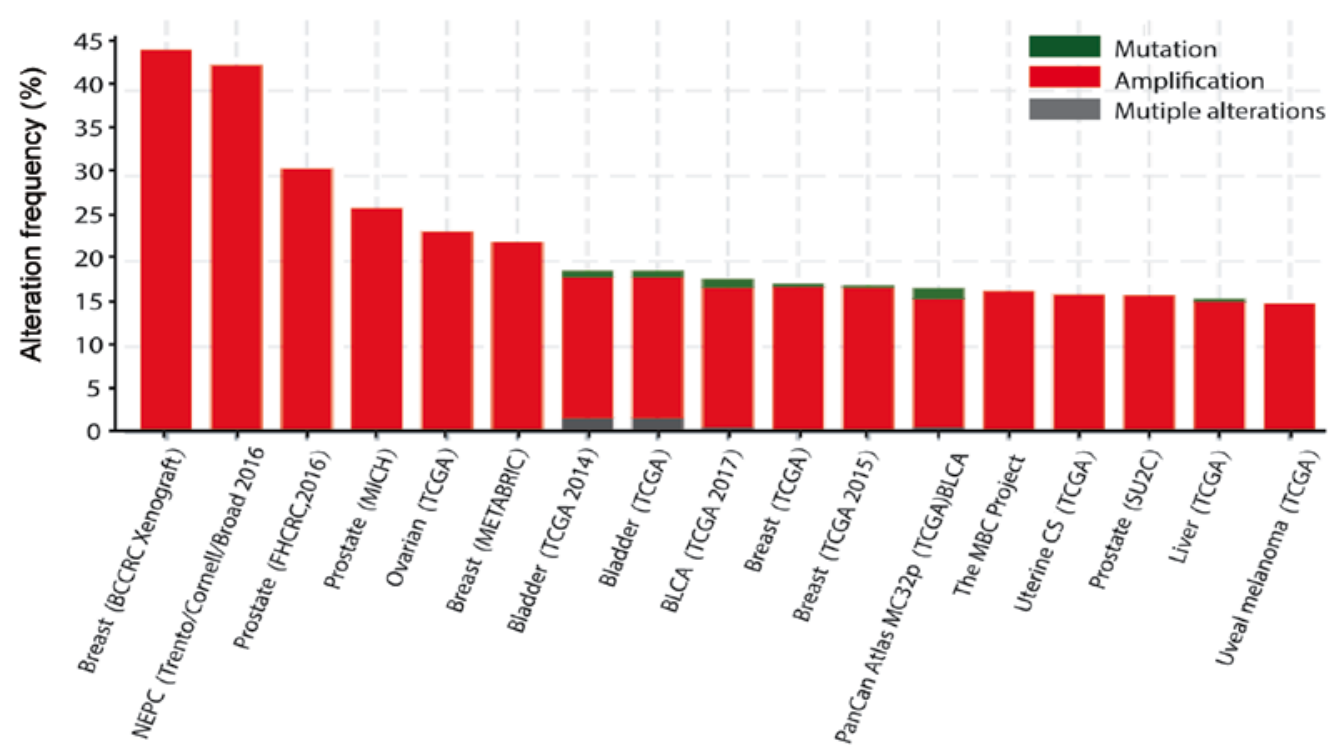

B

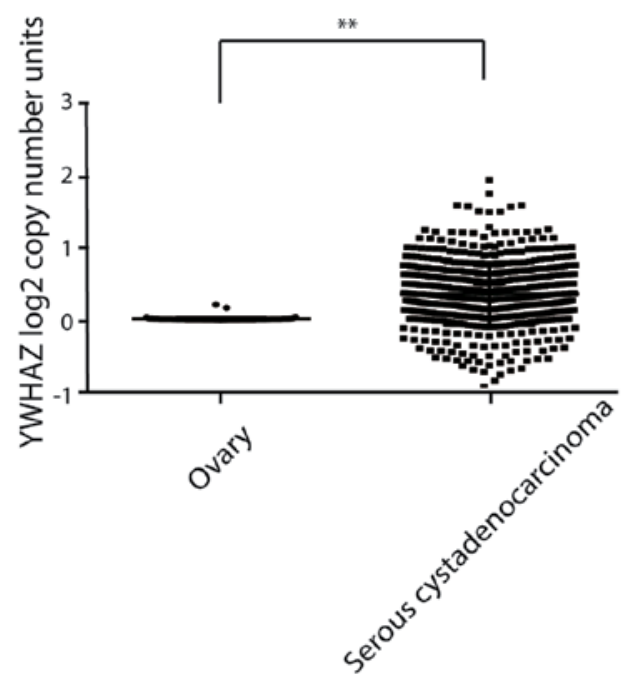

D
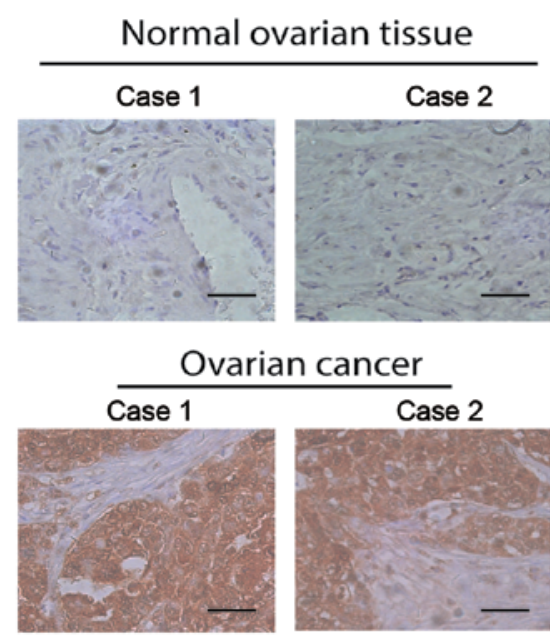

C

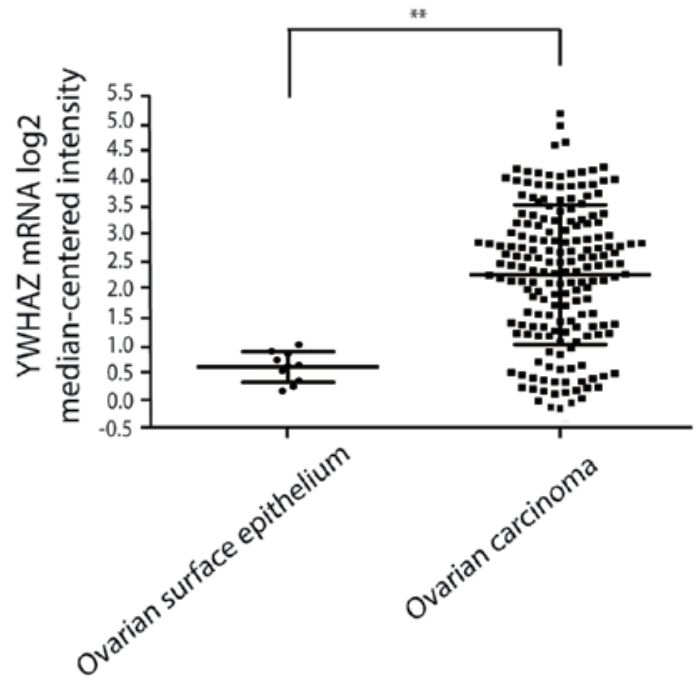

$\mathrm{E}$

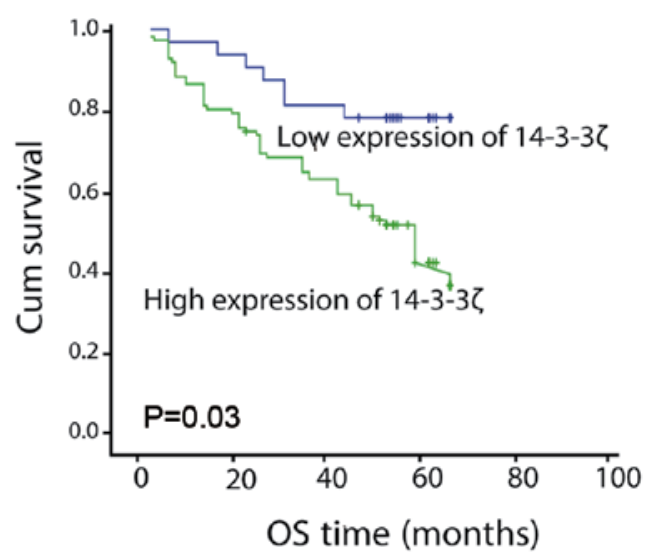

Figure 1. cBioportal and TCGA database analysis reveals that YWHAZ is highly expressed in ovarian cancer. (A) Cross-cancer alteration summary for YWHAZ from cBioPortal data. (B and C) Ovarian cancer tissue chip results in the TCGA library also revealed that YWHAZ expression in ovarian cancer tissues is significantly increased compared with normal ovarian tissues. (D) Immunohistochemical staining revealed YWHAZ expression in paraffin-embedded ovarian cancer tissues. Scale bar, $25 \mu \mathrm{m}$. (E) Cumulative survival curves based on YWHAZ expression. Patients with high YWHAZ expression had lower overall survival rates. ${ }^{* *} \mathrm{P}<0.01$. 
Table I. YWHAZ expression and clinicopathological parameters in 83 ovarian cancer cases.

\begin{tabular}{|c|c|c|c|c|}
\hline \multirow[b]{2}{*}{ Parameters } & \multirow[b]{2}{*}{ Total } & \multicolumn{2}{|c|}{ YWHAZ } & \multirow[b]{2}{*}{ P-value } \\
\hline & & Score <2 n (\%) & Score $\geq 2 \mathrm{n}(\%)$ & \\
\hline Ovarian cancer group & 83 & $32(38.6)$ & $51(61.4)$ & $0.000^{\mathrm{a}}$ \\
\hline Control group & 18 & $15(83.3)$ & $3(16.7)$ & \\
\hline Histologic subgroups & & & & 0.882 \\
\hline Serous & 60 & $14(23.3)$ & $46(76.7)$ & \\
\hline Endometriods & 11 & $5(45.4)$ & $6(54.6)$ & \\
\hline Clear cell & 12 & $5(41.7)$ & $7(58.3)$ & \\
\hline FIGO stage & & & & $0.026^{\mathrm{a}}$ \\
\hline I-II & 39 & $15(38.5)$ & $24(61.5)$ & \\
\hline III-IV & 44 & $4(9.1)$ & $40(90.9)$ & \\
\hline Lymph node status & & & & $0.017^{\mathrm{a}}$ \\
\hline Negative & 23 & $10(43.4)$ & $13(56.6)$ & \\
\hline Positive & 60 & $11(18.3)$ & $49(81.7)$ & \\
\hline Tumor size $(\mathrm{cm})$ & & & & $0.017^{\mathrm{a}}$ \\
\hline$<2$ & 32 & $15(43.3)$ & $17(56.7)$ & \\
\hline$\geq 2$ & 51 & $10(19.7)$ & $41(80.3)$ & \\
\hline
\end{tabular}

Statistical analyses were performed by Pearson $\chi^{2}$ test. ${ }^{\text {a }}<0.05$ was considered to indicate a statistically significant result. $\mathrm{P}$-values in bold emphasize statistical significance $(\mathrm{P}<0.001)$.

50 mM 2-deoxyglucose (2-DG; Sigma-Aldrich; Merck KGaA; D8375). Measurements were normalized to total protein content and reported as $\mathrm{mpH} / \mathrm{min}$ for ECAR. Each datum was determined in triplicate.

Clinical samples. For immunohistochemical staining of YWHAZ and analysis of its correlation with clinicopathological features, an ovarian cancer tissue microarray containing 83 cases of ovarian cancer samples and 18 cases of normal ovarian samples was used. These tissues were obtained from the Department of Gynecology, Obstetrics and Gynecology Hospital, Fudan University from May 2006 to January 2009. All human ovarian tissues were obtained with informed consent and all protocols were approved by the Ethics Review Committee of the Research Ethics Committee of the Fifth People's Hospital of Shanghai, Fudan University Hospital. Expression score was determined by staining intensity and immunoreactive cell percentage. Scoring was conducted according to the ratio and intensity of positive staining cells: $0-5 \%$ scored $0 ; 6-35 \%$ scored $1 ; 36-70 \%$ scored 2 ; and $>70 \%$ scored 3. The final score of YWHAZ expression was designated as low or high expression group as follows: Low expression, score 0-1; and high expression, score 2-3. All the scores of YWHAZ expression were performed in a blinded manner and determined independently by two senior pathologists.

Statistical analysis. All data were analyzed as the mean \pm standard deviation (SD). Statistical analyses were conducted using SPSS 14.0 software (SPSS Inc., Chicago, IL, USA). We performed the Pearson's $\chi^{2}$ test in cross tables to assess the relationships between the expression levels of YWHAZ and the clinicopathological factors. Overall survival (OS) was calculated using the Kaplan-Meier method. Comparison between groups was analyzed by one-way analysis of variance (ANOVA) followed by a post hoc test (Student-Newman-Keuls method). Values of $\mathrm{P}<0.05$ were considered to indicate a statistically significant result.

\section{Results}

YWHAZ is highly expressed in epithelial ovarian cancer tissues. To investigate the relationship between YWHAZ and the biological characteristics of ovarian cancer, we analyzed YWHAZ expression using the cBioPortal database (www.cbiopotal.org). The number of copies of the YWHAZ gene was increased in most tumors, such as breast, prostate and ovarian cancer (Fig. 1A). Using the TCGA database (http://cancergenome.nih.gov/), we analyzed YWHAZ copy number and mRNA levels in ovarian cancers compared with normal ovarian tissue. We found that YWHAZ mRNA expression and copy number in cancer tissues was similarly increased (Fig. 1B and C). Then, we performed an immunohistochemical (IHC) analysis of a tissue microarray that contained 83 epithelial ovarian cancer (EOC) tissue samples and 20 normal ovarian tissues. As shown in Fig. 1D, strong YWHAZ staining was observed in most EOC samples (61.4 and 51/83), whereas staining was minimal in normal ovarian tissues (16.7 and 3/18). Using Kaplan-Meier analysis, patients with high YWHAZ expression were found to be significantly associated with reduced overall survival (Fig. 1E).

Then, we analyzed the relevance of YWHAZ expression based on patient clinicopathological parameters. We found that patients with high YWHAZ expression exhibited higher tumor stage and worse prognosis (Table I). 
A

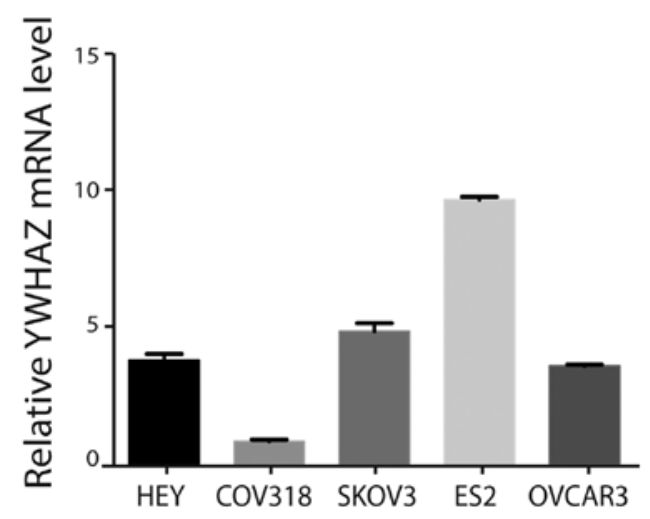

B

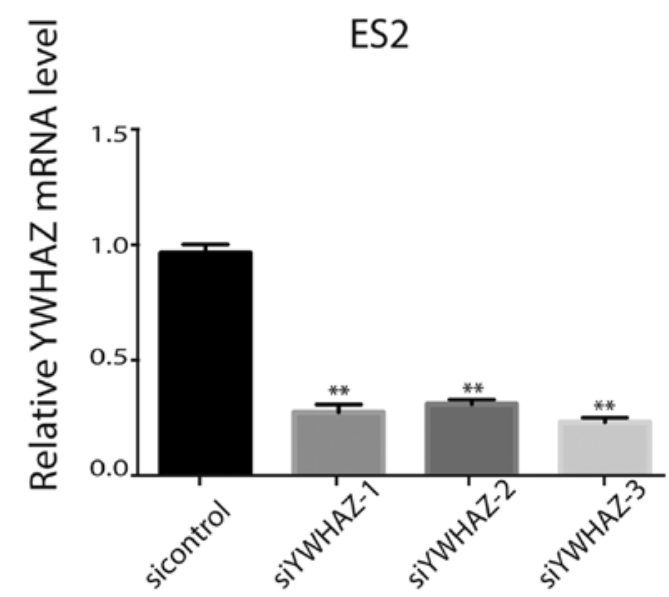

C

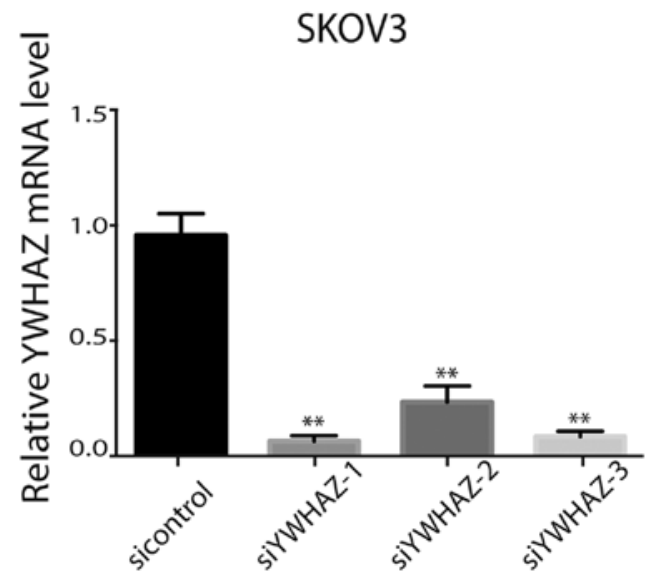

Figure 2. Expression and interference efficiency of YWHAZ in ovarian cancer cell lines. (A) YWHAZ mRNA expression in five ovarian cancer cell lines. (B and C) YWHAZ mRNA expression in (B) ES2 or (C) SKOV3 cells transfected with siRNAs against YWHAZ or scrambled RNAs as a negative control (NC) was analyzed by real-time PCR. ${ }^{* *} \mathrm{P}<0.01$.

YWHAZ expression in ES2 and SKOV3 cell lines transfected with siRNAs against YWHAZ. To select suitable cell lines for further research, we assessed YWHAZ expression in five ovarian cell lines (HEY, CAOV3, ES2, COV318 and SKOV3). The results showed that ES2 and SKOV3 cells that exhibit high metastatic potential expressed the highest levels of YWHAZ (Fig. 2A). Therefore, ES2 and SKOV3 cells were transfected with siRNAs targeting YWHAZ or a scrambled siRNA (labeled as control). RT-PCR and western blotting were used to validate the silencing effects of the siRNAs in the cell lines (Fig. 2B and C). The results verified that YWHAZ expression levels were significantly decreased by the three siRNAs against YWHAZ.

Silencing of YWHAZ suppresses ovarian cancer cell proliferation in vitro. Next, we investigated the effect of YWHAZ on cell viability. After transfection with siRNAs, the Cell Counting Kit-8 (CCK-8) assay was used to detect ES2 and SKOV3 proliferation. The results showed that ES2 and SKOV3 cell proliferation was suppressed by silencing of YWHAZ (Fig. 3A and B).

Silencing of $Y W H A Z$ promotes ovarian cancer cell apoptosis in vitro. To explore whether YWHAZ affects ovarian cancer cell apoptosis, ES2 and SKOV3 cells transfected with siRNAs against YWHAZ were used to detect cell apoptosis by flow cytometry. The results showed that YWHAZ silencing promoted ES2 and SKOV3 cell apoptosis (Fig. 3C and D).

Silencing of YWHAZ suppresses ovarian cancer cell migration in vitro and in vivo. To further study the functional role of YWHAZ in ovarian cancer, we investigated the effects of YWHAZ on ovarian cancer cell migration in vitro. Silencing of YWHAZ expression significantly inhibited ES2 and SKOV3 cell migration in vitro as assessed by Transwell migration assay (Fig. 4A and B), whereas YWHAZ overexpression promoted ES2 and SKOV3 cell migration in vitro (Fig. 4C and D) compared with the control group. Then, we constructed stable ES-2 and SKOV3 cell lines expressing shYWHAZ. The knockdown efficiency was determined by qRT-PCR and western blotting (Fig. 4E and F). Then, we injected these cells into the tail vein of BALB/C nude mice. Thirty-five days after injection, we examined tumor metastases in the mice by histological H\&E staining analyses. Compared with the control group, the number of metastatic lung nodules was significantly reduced in the interference group (Fig. 4G and $\mathrm{H}$ ). Consistent with our findings in vitro, genetic silencing of YWHAZ significantly reduced the tumor burden of EOC in the xenograft metastasis model in nude mice (Fig. 4G and $\mathrm{H}$ ).

Effects of the silencing of YWHAZ on glycolysis and the PI3K/AKT pathway. Considering that YWHAZ modulates cellular glycolysis in other cancers, we next investigated whether YWHAZ is involved in ovarian cancer glycolysis. First, we measured glycolysis-related enzymes upon YWHAZ silencing in ovarian cancer cells. In YWHAZ-silenced ES-2 and SKOV3 cells, the mRNA and protein expression levels of glycolysis-related enzymes, including lactate dehydrogenase A (LDHA), human platelet phosphofructokinase (PFKP) and glyceraldehyde-3-dehydrogenase (PGD), were significantly reduced (Fig. 5A and B). Then, we examined lactate production, extracellular acidification rates (ECAR) and NADH production to probe glycolysis. YWHAZ silencing significantly reduced lactate, ECAR and NADH production 
A

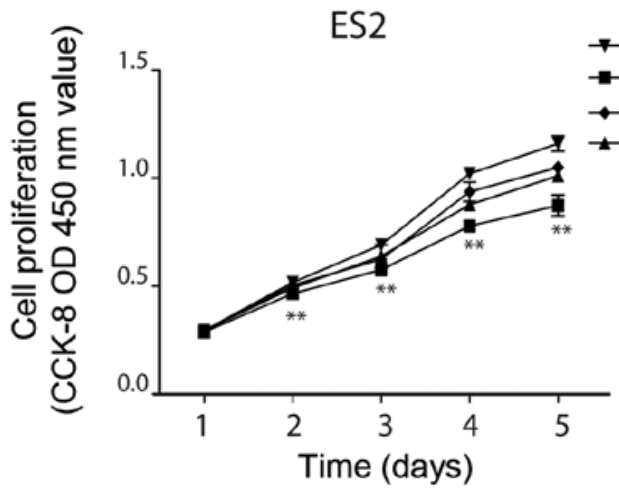

B

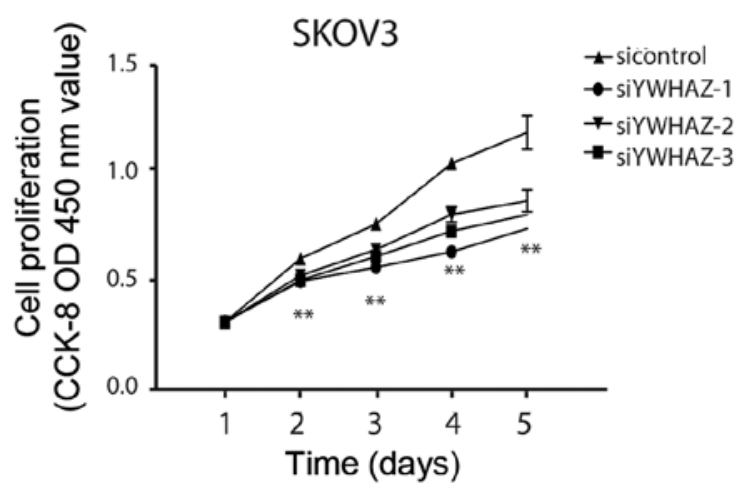

C

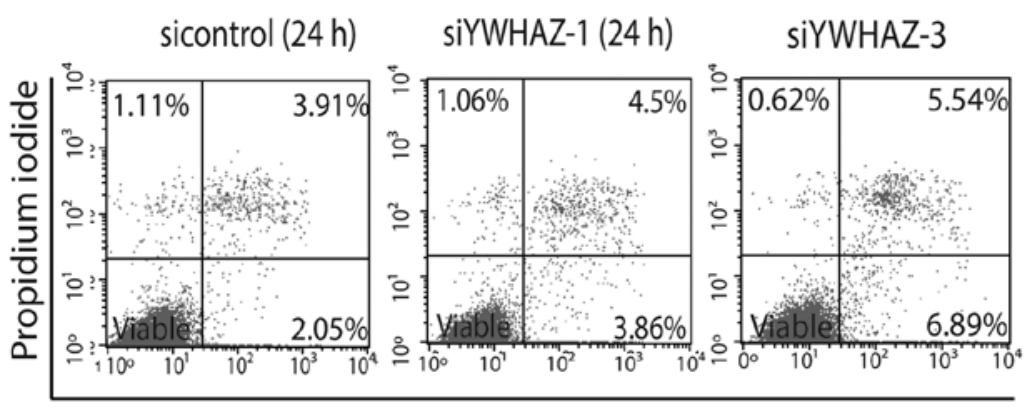

Annexin V-FITC

D

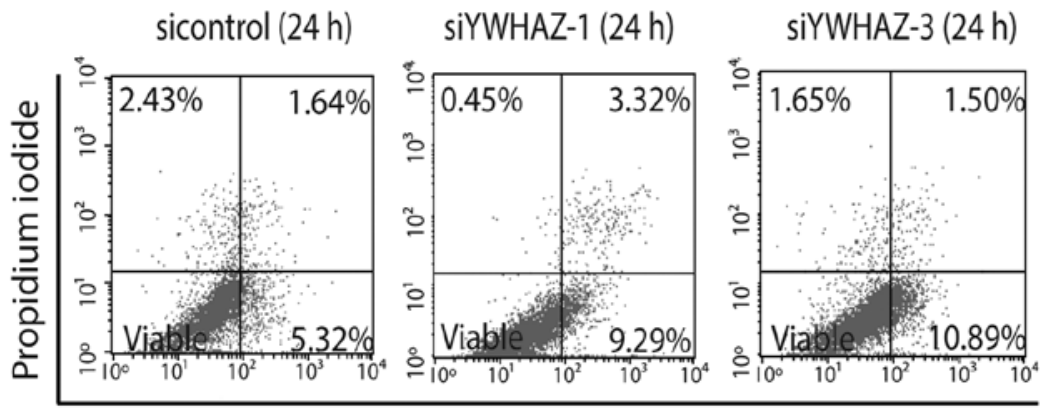

Annexin V-FITC
ES2

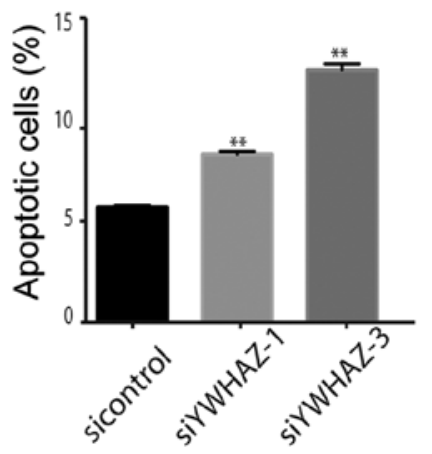

SKOV3

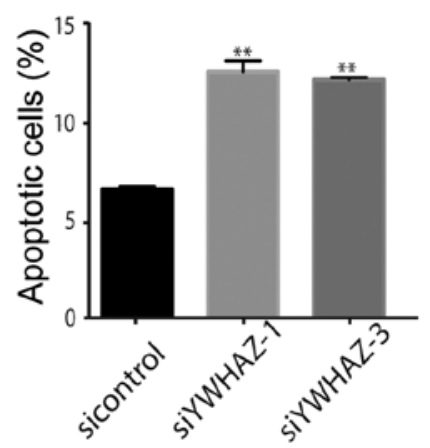

Figure 3. YWHAZ promotes EOC cell proliferation and inhibits EOC cell apoptosis in vitro. (A) ES2 and (B) SKOV3 cells transfected with siYWHAZ exhibited reduced cell viability as detected by CCK-8 assay. (C) ES2 and (D) SKOV3 cells transfected with siYWHAZ exhibited increased cell apoptosis. ${ }^{* *} \mathrm{P}<0.01$. EOC, epithelial ovarian cancer.

in ES-2 and SKOV3 cells (Fig. 5C and D). These data suggest that YWHAZ can promote ovarian cancer cell glycolysis by regulating the expression of related metabolic enzymes. YWHAZ binds numerous effectors with a phosphoserine or phosphothreonine motif and participates in cancer development and progression by regulating a large spectrum of both general and specialized signaling pathways. Importantly, YWHAZ interacts with Akt1 (22), a crucial factor involved in the regulation of Warburg effect by targeting glycolytic enzymes (6). This information prompted us to explore the possibility that YWHAZ may stimulate the Warburg effect through its regulation of Akt1 in EOC cells. As shown in Fig. 5E, levels of phosphorylated Akt1 were reduced after genetic inhibition of YWHAZ. In contrast, YWHAZ overexpression upregulated Akt1 phosphorylation (Fig. 5F). Notably, YWHAZ also regulated the phosphorylation of PI3K (Fig. 5F), suggesting that YWHAZ also affected Akt1 through PI3K signaling. Furthermore, the expression level of vimentin, a downstream substrate of Akt1, was downregulated by YWHAZ silencing and upregulated by YWHAZ overexpression. These data suggest that YWHAZ promotes the Warburg effect by modulating PI3K/Akt1 signaling.

Collectively, these results demonstrated that YWHAZ promotes ovarian cancer growth and metastasis, which is probably mediated by regulating PI3K/Akt1/vimentin signaling and glycolysis. 
A

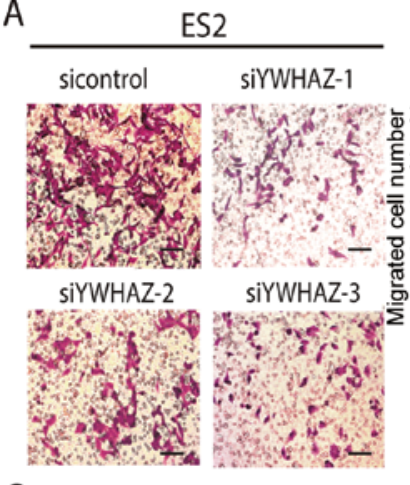

C
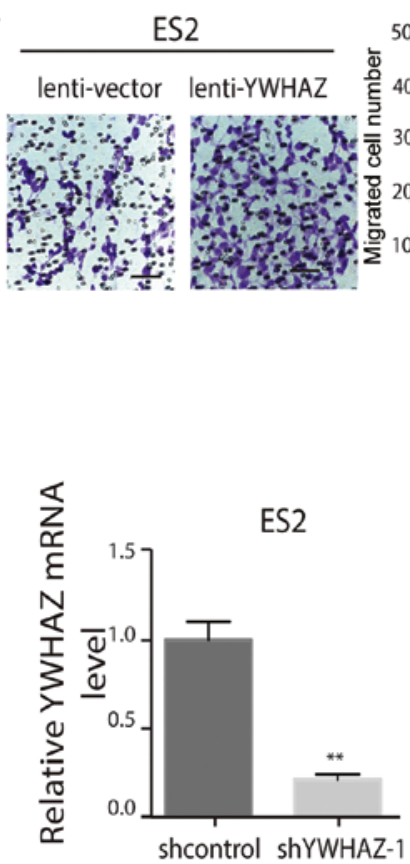

YWHAZ

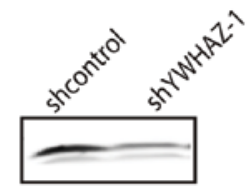

Tubulin

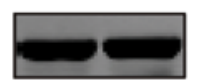

G

ES2

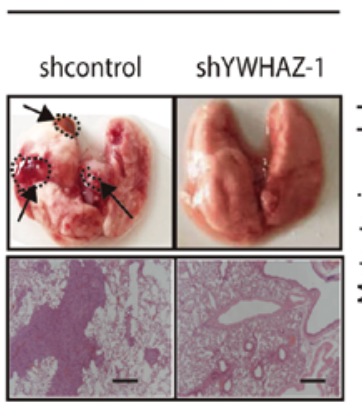

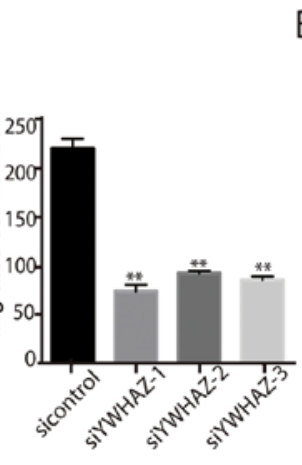

B

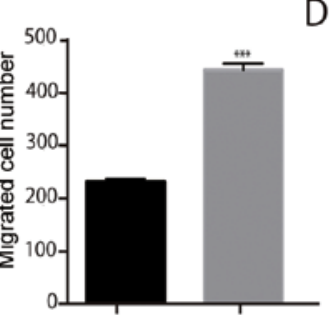

D

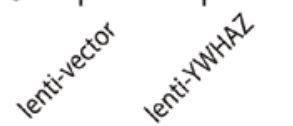

SKOV3
sicontrol siYWHAZ-1
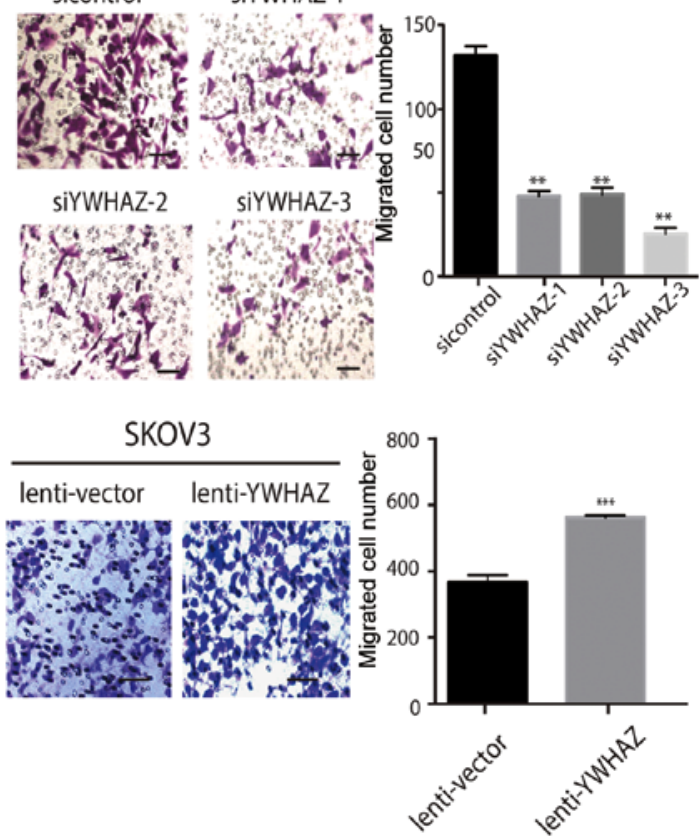

$\mathrm{F}$

YWHAZ

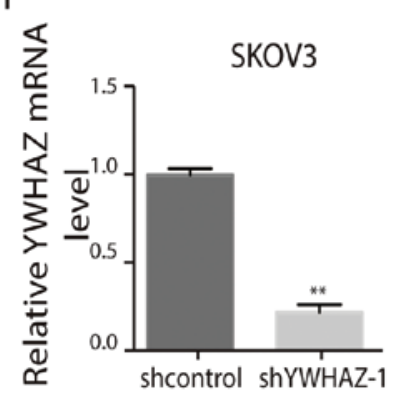

Tubulin

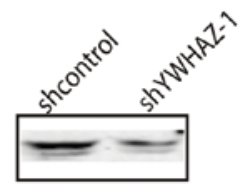

$\mathrm{H}$
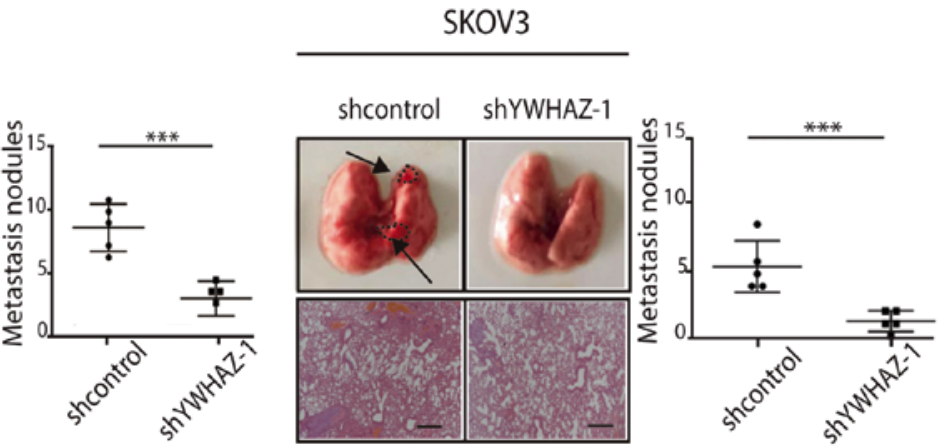

Figure 4. YWHAZ promotes EOC cell migration in vitro and in vivo. (A and B) Transwell migration assays using ES2 and SKOV3 cells transfected with Lenti-shYWHAZ-(1, 2, 3) or NC (sicontrol). Scale bar, $100 \mu \mathrm{m}$. (C and D) Transwell migration assays using ES-2 and SKOV3 cells with overexpression of YWHAZ. Representative images are shown on the left, and the quantification of 3 randomly selected fields is shown on the right (original magnification, $\mathrm{x} 200$ ). The results shown are mean \pm SD. Scale bar, $100 \mu \mathrm{m}$. (E and F) YWHAZ mRNA and protein expression levels after YWHAZ gene silencing in ES2 and SKOV3. shcontrol, lenti-shcontrol; shYWHAZ-1, lenti-shYWHAZ-1. (G and H) Representative images of collected lungs and hematoxylin and eosin staining of lung tissues in control and YWHAZ-interference groups are shown on the left. Numbers of lung metastatic nodules were counted and the results are shown on the right. shcontrol, lenti-shcontrol; shYWHAZ-1, lenti-shYWHAZ-1. Values are means means $\pm \mathrm{SD}$. ${ }^{* *} \mathrm{P}<0.01$ and ${ }^{* * *} \mathrm{P}<0.001$. Scale bar, $100 \mu \mathrm{m}$. EOC, epithelial ovarian cancer. 
A

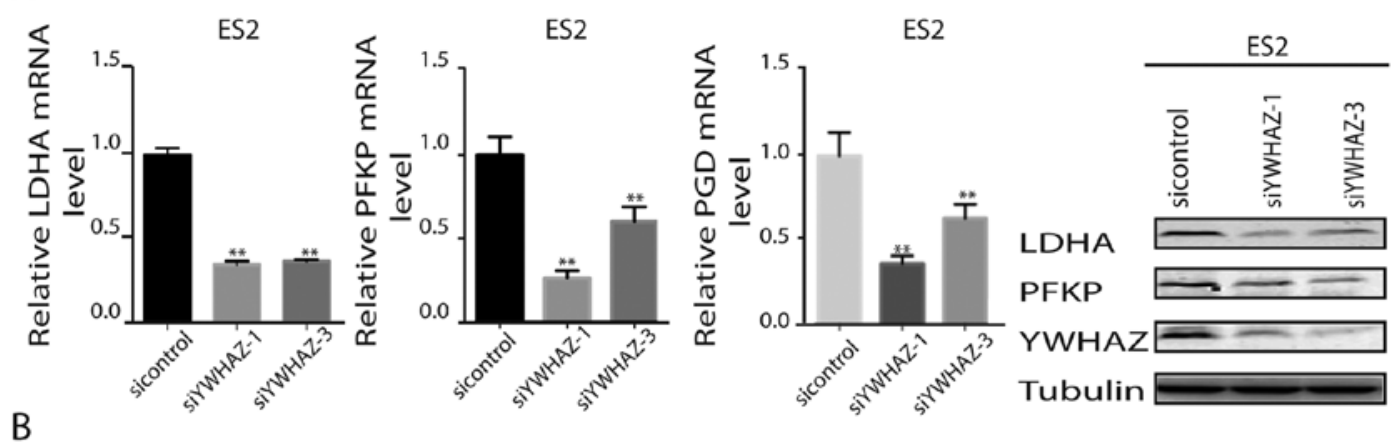

B

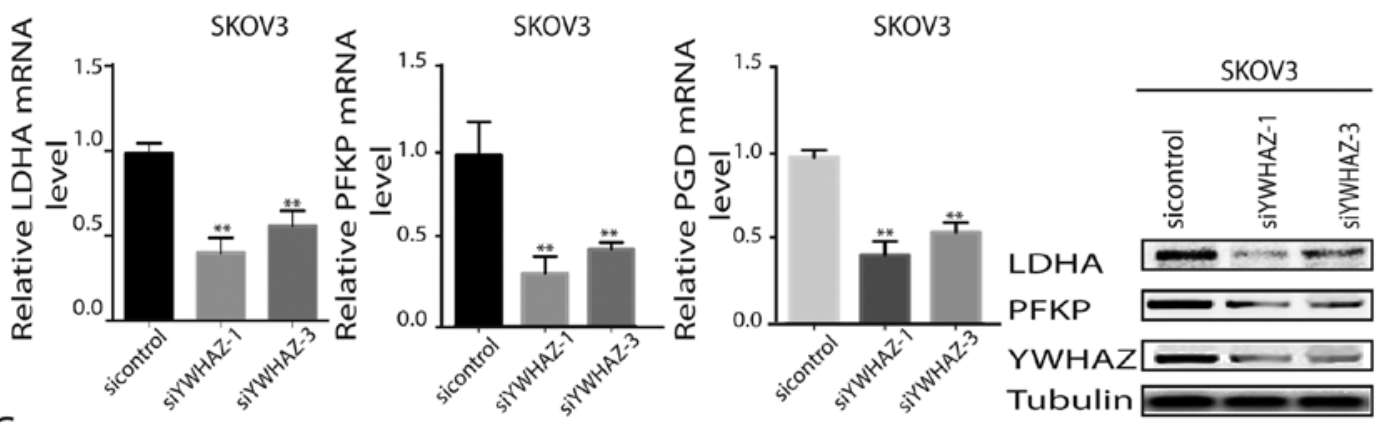

C
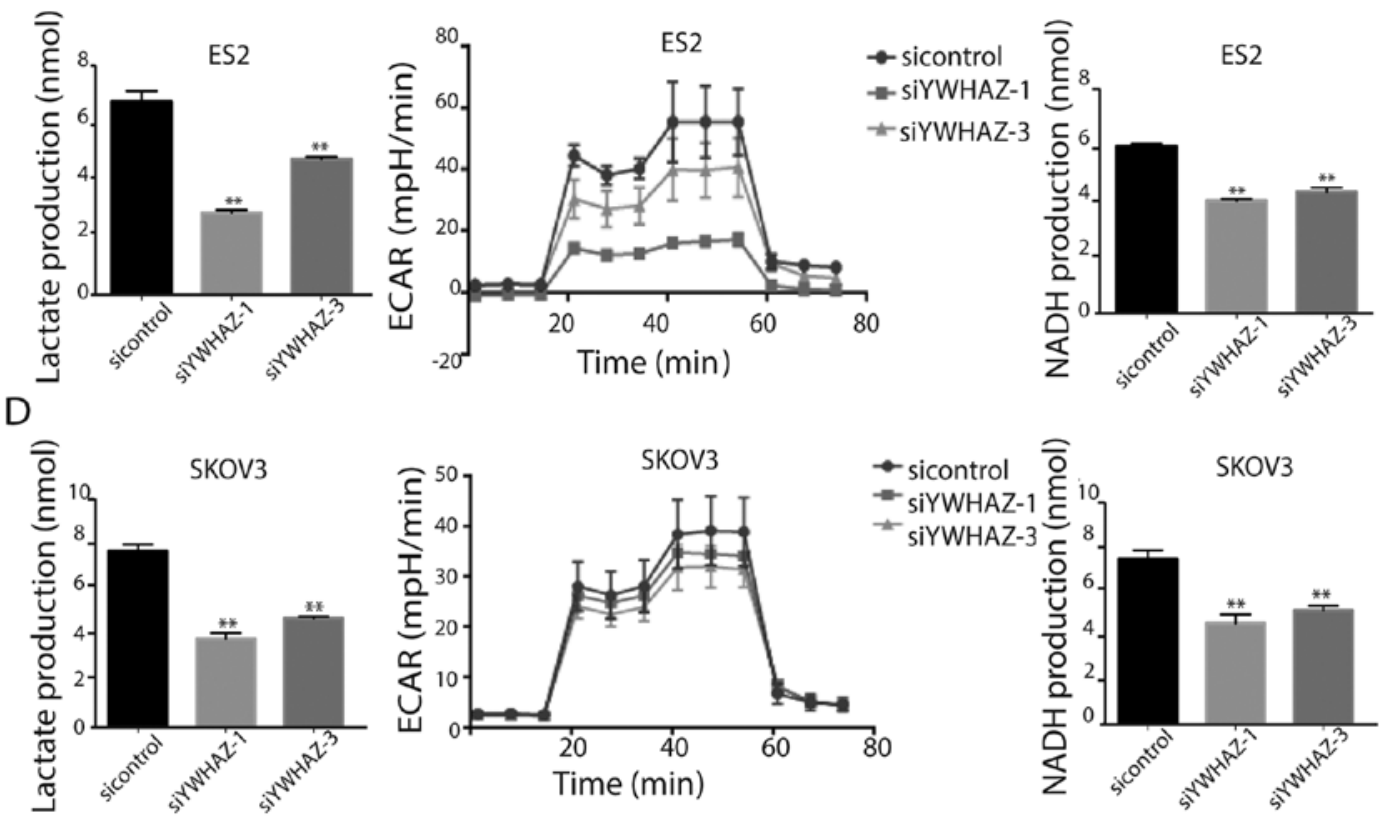

$\mathrm{E}$

$\mathrm{F}$
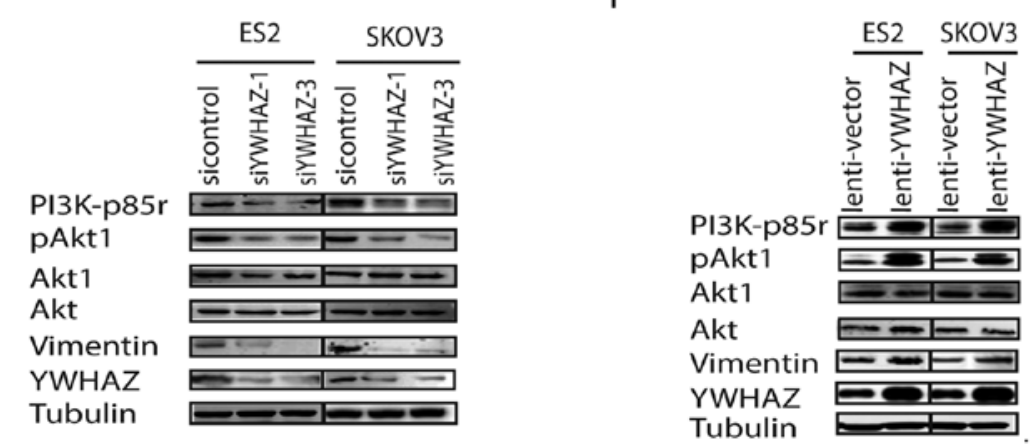

Figure 5. Effect of YWHAZ on glycolysis metabolism in ovarian cancer cells. (A and B) The mRNA and protein expression of related glycolytic enzymes [lactate dehydrogenase A (LDHA), human platelet phosphofructokinase (PFKP) and glyceraldehyde-3-dehydrogenase (PGD)] was examined after (A) ES-2 and (B) SKOV3 cells were transfected with siRNAs targeting YWHAZ by real-time PCR and western blot analysis. (C and D) The expression of glycolytic metabolites [lactate production, extracellular acidification rate (ECAR) and nicotinamide adenine dinucleotide (NADH) production] after (C) ES-2 and (D) SKOV3 cells were transfected with siRNAs targeting YWHAZ. (E and F) The protein expression of PI3K, pAkt1 and vimentin were detected after ES-2 and SKOV3 cells were transfected with (E) siRNAs targeting YWHAZ or (F) lenti-YWHAZ overexpressing plasmid. Tubulin was used as an internal control. Values are means mean $\pm \mathrm{SD}$. ${ }^{* *} \mathrm{P}<0.01$. 


\section{Discussion}

Epithelial ovarian cancer (EOC) is the most lethal of all gynecological malignancies. It is typically diagnosed at a late stage, with a 5-year survival rate of less than $30 \%$. Metastasis plays a crucial role in promoting ovarian tumor progression and decreasing patient survival rates (23). In addition, it is considered that the development of ovarian cancer is related to diverse factors including abnormality of chromosome, activation of oncogenes, inactivation of tumor-suppressor genes and apoptosis inhibition (24). However, the underlying mechanisms by which ovarian cancer spreads have yet to be thoroughly explored. Thus, it is critical to understand the molecular mechanisms that promote the metastasis and progression in EOC. In the present study, we aimed to address the contributions of YWHAZ in EOC and explore its potential diagnostic and therapeutic value.

Metabolic alterations have been suggested to play a crucial role in cancer development. The key metabolic changes in cancer include aerobic glycolysis and macromolecular synthesis, causing anti-apoptosis in cancer cells. Activation of Akt enhances glycolytic activity and is commonly observed in cancer cells, including ovarian cancer. Considering the high incidence of chemoresistance and the rapid spread of disease in ovarian cancer, metabolic approaches represent rational strategies to improve the poor prognosis of ovarian cancer.

The 14-3-3 $\xi$ gene, which is also known as YWHAZ on $8 \mathrm{q} 22$, is often amplified in numerous tumor types, such as breast and prostate cancer $(15,25)$, and high expression of $14-3-3 \zeta$ in the primary tumor is significantly associated with earlier time to recurrence and with distant metastasis (26). We examined YWHAZ expression in EOC and this study demonstrated that the YWHAZ-positive tumor rate was significantly increased in EOC. We analyzed the correlation between YWHAZ expression and ovarian cancer clinicopathological parameters and found that YWHAZ expression levels were closely related to tumor stage, tumor size and metastasis. Kaplan-Meier analysis revealed that ovarian cancer patients with YWHAZ-positive tumors had shorter overall survival than YWHAZ-negative patients. Therefore, we identified that YWHAZ may represent a potential predictor of poor prognosis in ovarian cancer patients, which is consistent with a previous study (26).

Upregulation of YWHAZ is very common in many aggressive human types of cancers as it is linked to tumor cell proliferation and migration. In this study, when YWHAZ was silenced, the proliferation and migration of the EOC cell lines were significantly inhibited, whereas the apoptosis of the EOC cell lines was promoted. YWHAZ/14-3-3 integrates numerous signaling pathways by activating the MEK-ERK-CREB axis to promote early breast cancer transformation (7); playing a crucial role during leukemogenesis through the c-Myc/miR-451/YWHAZ/AKT cascade (27); promoting dynamic interactions with the core autophagy regulator Atg9A to modulate autophagy (28); by activating Rac1-GTPase to enhance prostate cancer cell-matrix interactions, motility and transendothelial migration (29); by activating JNK/p38/MAPK signaling pathway to enhance the anticancer effect of $c i s$-diammine dichloroplatinum (CDDP) in hepatoma cell lines (30); by activating the TGF $\beta / S m a d$ pathway leading to ZFHX1B/SIP-1 upregulation, E-cadherin loss and epithelial-mesenchymal transition (EMT) reduced cell adhesion (15); and reducing receptor tyrosine kinase (HER2 and EGFR) signaling (HER2/EGFR) to reverse endocrine resistance in breast cancer (16).

Although the functional roles of YWHAZ in tumor cell proliferation, migration and apoptosis have been well established, the underlying mechanisms by which YWHAZ promotes ovarian cancer cell proliferation, migration and apoptosis inhibition remain unclear. From a therapeutic perspective, targeting YWHAZ is of particular interest as the present study revealed increased YWHAZ expression in EOC which was found to be associated with poor prognosis, consistent with the results of $\mathrm{He}$ et al (26).

Tumor cells often exhibit an altered metabolic phenotype. As ovarian cancer progresses, complete oxidation of glucose and fatty acids is significantly decreased and occurs concurrently with increases in lactate excretion and $3 \mathrm{H}$-deoxyglucose uptake by late-stage cancer cells, shifting the cells towards a more glycolytic phenotype, confirming the metabolic changes during ovarian cancer progression (8). An altered metabolism during ovarian cancer progression allows for increased macromolecular synthesis and unrestrained growth of the tumor-initiating cell population or cancer stem cells (CSCs/TICs). Ovarian cancer CSCs/TICs exhibit reductions in glucose and fatty acid oxidation with a concomitant increase in lactate secretion (31). In recent years, mounting evidence indicates that numerous multifaceted factors are involved in glycolysis. Altered energy metabolism has been confirmed to be as widespread in cancer cells as many of the other cancer-associated traits that have been accepted as hallmarks of cancer (4). These results suggest that the therapeutic targets in the glycolysis pathway could be utilized as anticancer strategies.

Studies have confirmed that YHWHAZ participates in glucose metabolism. YWHAZ can protect $\beta$ cells from multiple stresses by preventing BAD-BAX mitochondrial localization (18). YWHAZ gene knockout mice (14-3-3६KO) exhibited elevated fasting insulin levels while maintaining normal $\beta$ cell responsiveness to glucose when compared with wild-type littermate controls (32). As a response to intratumoral hypoxia, HIF-1 $\alpha$ also mediates metabolic alterations that drive cancer progression (22). Under hypoxic conditions in vitro, 14-3-3 5 knockdown inhibits hypoxia-induced HCC invasion by the HIF-1 $\alpha /$ EMT pathway (14).

In addition, YWHAZ expression is strongly correlated with the expression of canonical glycolytic genes, particularly lactate dehydrogenase A (LDHA). Thus, we aimed to ascertain whether downregulation of YWHAZ could influence glycolysis in ovarian cancer. Our results showed that upon silencing of YWHAZ, the expression of glycolysis metabolites (lactate, ECAR and NADH) and enzymes (LDHA) were decreased, which is consistent with Chang et al (7).

Existing data indicated that PI3K/Akt1 participates in glycolysis (33). In this study, we found that phosphorylation of Akt1 and its upstream kinase PI3K was reduced after YWHAZ knockdown in ovarian cancer cells. Notably, Akt1 phosphorylates Ser-58 on YWHAZ both in vitro and in intact cells (22). Moreover, the Ser-58 phosphorylated form inhibits its interaction with TP53 and p53 transcriptional activity (34). 
Collectively, we can infer that YWHAZ is a substrate of Akt1 and that YWHAZ promotes PI3K/Akt1/vimentin signaling, forming a feedback functional loop to promote cancer glycolysis, proliferation and migration. An important issue that needs future investigation is how YWHAZ regulates PI3K/Akt1 signaling. One possibility is by interacting with an upstream factor with a phosphoserine or phosphothreonine motif.

In this study, we determined that high YWHAZ expression was associated with poor prognosis in ovarian cancer. Regarding the biological significance of YWHAZ, it promoted proliferation and migration of ovarian cancer cells. Additionally, the silencing of YWHAZ reduced the glycolytic capability of EOC cells. Investigation into the molecular mechanisms revealed that YWHAZ silencing downregulated the phosphorylation of PI3K/Akt1 in EOC cell lines. Taken together, our results suggest a role for YWHAZ in the progression of ovarian cancer and identify YWHAZ as a potentially important molecule for human ovarian cancer progression. Further research is needed to clarify which signaling pathway is involved in YWHAZ-mediated regulation of PI3K/Akt1 expression, thereby affecting glycolysis in ovarian cancer cells and promoting cell proliferation and metastasis.

\section{Acknowledgements}

We thank Dr Lei Zhu, Dr Jun Li and Dr Ya-Hui Wang for technical help and advice.

\section{Funding}

The present study was supported by the Natural Science Foundation, Minhang, Shanghai (no. 2016MHZ02).

\section{Availability of data and materials}

The datasets used and/or analyzed during the current study are available from the corresponding author on reasonable request.

\section{Authors' contributions}

XMY and LWZ conceived and designed the study, analyzed and interpreted the data, and provided a critical review of the manuscript. JS and JY collected and analyzed the data and wrote the manuscript. JS, JY, SHJ, HF, YPC and ZYW prepared the experimental materials and performed the in vitro assays. SHJ and ZYW performed the in vivo assays. All authors read and approved the manuscript and agree to be accountable for all aspects of the research in ensuring that the accuracy or integrity of any part of the work are appropriately investigated and resolved.

\section{Ethics approval and consent to participate}

All experimental protocols were approved by the Institutional Review Board of the Department of Laboratory Animal Science of Fudan University (Shanghai, China).

\section{Patient consent for publication}

Not applicable.

\section{Competing interests}

The authors declare that they have no competing interests.

\section{References}

1. Siegel RL, Miller KD and Jemal A: Cancer statistics, 2018. CA Cancer J Clin 68: 7-30, 2018.

2. Baldwin LA, Huang B, Miller RW, Tucker T, Goodrich ST, Podzielinski I, DeSimone CP, Ueland FR, van Nagell JR and Seamon LG: Ten-year relative survival for epithelial ovarian cancer. Obstet Gynecol 120: 612-618, 2012.

3. Damia G and Sessa C: Successes and limitations of targeted cancer therapy in ovarian cancer. Prog Tumor Res 41: 89-97, 2014.

4. Hanahan D and Weinberg RA: Hallmarks of cancer: The next generation. Cell 144: 646-674, 2011.

5. Hu S, Balakrishnan A, Bok RA, Anderton B, Larson PE, Nelson SJ, Kurhanewicz J, Vigneron DB and Goga A: 13C-pyruvate imaging reveals alterations in glycolysis that precede c-MYC induced tumor formation and regression. Cell Metab 14: 131-142, 2011.

6. Jiang SH, Li J, Dong FY, Yang JY, Liu DJ, Yang XM, Wang YH, Yang MW, Fu XL, Zhang XX, et al: Increased serotonin signaling contributes to the Warburg effect in pancreatic tumor cells under metabolic stress and promotes growth of pancreatic tumors in mice. Gastroenterology 153: 277-291.e19, 2017.

7. Chang CC, Zhang C, Zhang Q, Sahin O, Wang H, Xu J, Xiao Y, Zhang J, Rehman SK, Li P, et al: Upregulation of lactate dehydrogenase a by 14-3-3 $\zeta$ leads to increased glycolysis critical for breast cancer initiation and progression. Oncotarget 7: 35270-35283, 2016.

8. Slupsky CM, Steed H, Wells TH, Dabbs K, Schepansky A, Capstick V, Faught W and Sawyer MB: Urine metabolite analysis offers potential early diagnosis of ovarian and breast cancers. Clin Cancer Res 16: 5835-5841, 2010.

9. Anderson AS, Roberts PC, Frisard MI, McMillan RP, Brown TJ, Lawless MH, Hulver MW and Schmelz EM: Metabolic changes during ovarian cancer progression as targets for sphingosine treatment. Exp Cell Res 19: 1431-1442, 2013.

10. Sun L, Yin Y, Clark LH, Sun W, Sullivan SA, Tran AQ, Han J, Zhang L, Guo H, Madugu E, et al: Dual inhibition of glycolysis and glutaminolysis as a therapeutic strategy in the treatment of ovarian cancer. Oncotarget 8: 63551-63561, 2017.

11. Watanabe N, Komatsu S, Ichikawa D, Miyamae M, Ohashi T, Okajima W, Kosuga T, Konishi H, Shiozaki A, Fujiwara H, et al: Overexpression of YWHAZ as an independent prognostic factor in adenocarcinoma of the esophago-gastric junction. Am J Cancer Res 6: 2729-2736, 2016.

12. Neal CL, Yao J, Yang W, Zhou X, Nguyen NT, Lu J, Danes CG, Guo H, Lan KH, Ensor J, et al: 14-3-3zeta overexpression defines high risk for breast cancer recurrence and promotes cancer cell survival. Cancer Res 69: 3425-3432, 2009.

13. Lin M, Morrison CD, Jones S, Mohamed N, Bacher J and Plass C: Copy number gain and oncogenic activity of YWHAZ/14-3-3zeta in head and neck squamous cell carcinoma. Int J Cancer 125: 603-611, 2009.

14. Tang Y, Liu S, Li N, Guo W, Shi J, Yu H, Zhang L, Wang K, Liu S and Cheng S: 14-3-3̧ promotes hepatocellular carcinoma venous metastasis by modulating hypoxia-inducible factor- $1 \alpha$. Oncotarget 7: 15854-15867, 2016.

15. Lu J, Guo H, Treekitkarnmongkol W, Li P, Zhang J, Shi B, Ling C, Zhou X, Chen T, Chiao PJ, et al: 14-3-3zeta cooperates with ErbB2 to promote ductal carcinoma in situ progression to invasive breast cancer by inducing epithelial-mesenchymal transition. Cancer Cell 16: 195-207, 2009.

16. Bergamaschi A, Frasor J, Borgen K, Stanculescu A, Johnson P, Rowland K, Wiley EL and Katzenellenbogen BS: $14-3-3 \zeta$ as a predictor of early time to recurrence and distant metastasis in hormone receptor-positive and -negative breast cancers. Breast Cancer Res Treat 137: 689-696, 2013.

17. Pozuelo Rubio M, Peggie M, Wong BH, Morrice $\mathrm{N}$ and MacKintosh C: 14-3-3s regulate fructose-2,6-bisphosphate levels by binding to PKB-phosphorylated cardiac fructose-2,6-bisphosphate kinase/phosphatase. EMBO J 2: 3514-3523, 2003.

18. Lim GE, Piske M and Johnson JD: 14-3-3 proteins are essential signaling hubs for beta cell survival. Diabetologia 56: 825-837, 2013. 
19. Kim CY, Jeong SY, Chong GO, Son SH, Jung JH, Kim DH, Lee SW, Ahn BC and Lee J: Quantitative metabolic parameters measured on F-18 FDG PET/CT predict survival after relapse in patients with relapsed epithelial ovarian cancer. Gynecol Oncol 136: 498-504, 2015.

20. Livak KJ and Schmittgen TD: Analysis of relative gene expression data using real-time quantitative PCR and the 2(-Delta Delta C(T)) method. Methods 25: 402-408, 2001.

21. Yang XM, Cao XY, He P, Li J, Feng MX, Zhang YL, Zhang XL, Wang YH, Yang Q, Zhu L, et al: Overexpression of Rac GTPase activating protein 1 contributes to proliferation of cancer cells by reducing hippo signaling to promote cytokinesis. Gastroenterology 155: 1233-1249.e22, 2018.

22. Powell DW, Rane MJ, Chen Q, Singh S and McLeish KR: Identification of 14-3-3zeta as a protein kinase B/Akt substrate. J Biol Chem 277: 21639-21642, 2002.

23. Yeung TL, Leung CS, Yip KP, Au Yeung CL, Wong ST and Mok SC: Cellular and molecular processes in ovarian cancer metastasis. A review in the theme: Cell and molecular processes in cancer metastasis. Am J Physiol Cell Physiol 309: C444-C456, 2015.

24. VaughanS,CowardJI,BastRCJr,Berchuck A,BerekJS,BrentonJD, Coukos G, Crum CC, Drapkin R, Etemadmoghadam D, et al: Rethinking ovarian cancer: Recommendations for improving outcomes. Nat Rev Cancer 11: 719-725, 2011.

25. Murata T, Takayama K, Urano T, Fujimura T, Ashikari D, Obinata D, Horie-Inoue K, Takahashi S, Ouchi Y, Homma Y and Inoue S: 14-3-3 upregulated in prostate cancer and promotes prostate cancer cell proliferation and survival. Clin Cancer Res 18: 5617-5627, 2012.

26. He Y, Wu X, Liu X, Yan G and Xu C: LC-MS/MS analysis of ovarian cancer metastasis-related proteins using a nude mouse model: 14-3-3 zeta as a candidate biomarker. J Proteome Res 9: $6180-6190,2010$.
27. Su R, Gong JN, Chen MT, Song L, Shen C, Zhang XH, Yin XL, Ning HM, Liu B, Wang F, et al: c-Myc suppresses

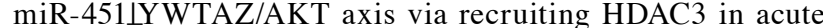
myeloid leukemia. Oncotarget 7: 77430-77443, 2016.

28. Weerasekara VK, Panek DJ, Broadbent DG, Mortenson JB, Mathis AD, Logan GN, Prince JT, Thomson DM, Thompson JW and Andersen JL: Metabolic-stress-induced rearrangement of the 14-3-3 $\zeta$ interactome promotes autophagy via a ULK1- and AMPK-regulated 14-3-3\} interaction with phosphorylated Atg9. Mol Cell Biol 34: 4379-4388, 2014.

29. Goc A, Abdalla M, Al-Azayzih A and Somanath PR: Rac1 activation driven by 14-3-3 $\zeta$ dimerization promotes prostate cancer cell-matrix interactions, motility and transendothelial migration. PLoS One 7: e40594, 2012.

30. Choi JE, Hur W, Jung CK, Piao LS, Lyoo K, Hong SW, Kim SW, Yoon HY and Yoon SK: Silencing of 14-3-3 $\zeta$ over-expression in hepatocellular carcinoma inhibits tumor growth and enhances chemosensitivity to cis-diammined dichloridoplatium. Cancer Lett 303: 99-107, 2011.

31. Anderson AS, Roberts PC, Frisard MI, Hulver MW and Schmelz EM: Ovarian tumor-initiating cells display a flexible metabolism. Exp Cell Res 328: 44-57, 2014.

32. Lim GE, Piske M, Lulo JE, Ramshaw HS, Lopez AF and Johnson JD: Ywhaz/14-3-3\} deletion improves glucose tolerance through a GLP-1-dependent mechanism. Endocrinology 157: 2649-2659, 2016.

33. Taylor C, Mannion D, Miranda F, Karaminejadranjbar M, Herrero-Gonzalez S, Hellner K, Zheng Y, Bartholomeusz G, Bast RC Jr and Ahmed AA: Loss of PFKFB4 induces cell death in mitotically arrested ovarian cancer cells. Oncotarget 8 : 17960-17980, 2017.

34. Danes CG, Wyszomierski SL, Lu J, Neal CL, Yang W and Yu D: 14-3-3 zeta down-regulates p53 in mammary epithelial cells and confers luminal filling. Cancer Res 68: 1760-1767, 2008. 